\title{
Classification of Transmission Line Faults Using
}

\section{Wavelet Transform}

\author{
Sandhya.B ${ }^{1}$, Dinesh shetty ${ }^{2}$ \\ PG Scholar, NMAMIT, Nitte, Karkala, India ${ }^{1}$ \\ Assistant Professor, NMAMIT, Nitte, Karkala, India ${ }^{2}$
}

\begin{abstract}
Wavelet transform technique is a robust and versatile method to analyze non-stationary,non-periodic wide band signal such as transient signal. Wavelet transform which is new tool for caballing both time and frequency information simultaneously. The suitable wavelet for analysing power system transients is Daubechies( $\mathrm{Db})$ wavelets. This paper focused on transmission line faults classification using wavelet analysis. The different faults in the transmission line (TL) are detected and classified using fault classification algorithm based on wavelet MRA(Multi resolution technique) technique.
\end{abstract}

Keywords: DWT, CWT, MRA,WT, TL.

\section{INTRODUCTION}

A transmission line establishes the major part of power system. Transmission lines runs over thousands of kilometres to supply electricity to the consumers. So large number of fault may occur in the transmission line which causes irregularity in the power flow through the lines. Basically a fault occurs when two or more conductor comes in contact with each other or with ground. When they exposed to atmosphere, chance of occurrence of fault in transmission line is very high which has to be minimized immediately in order to reduce damage caused by it. The first step involved in the power system relaying algorithm is to detect and classification of faults. The fault in the transmission line is usually classified as line (L) to ground $(\mathrm{G})$, double line to ground and three phase faults. For signal analysis, "wavelet transform" the most effective mathematical tools used. The wavelet analysis and wavelet transform is emerged into powerful tool for signal processing and its applications. Wavelet analysis is very efficient when the analysed signal has transients. The advantage of WT( wavelet transform) over STFT( short time Fourier transform) is that the short Fourier analysis which uses single analysis window(short/long), the wavelet uses short window at high frequency and the long window at low frequency.

\section{WAVELET TRANSFORM}

Wavelet means small wave. Analyse the signal with short duration having finite energy function is called wavelet analysis. We manipulate wavelet into two ways. First one is scaling. The second one is translation. Wavelet transformation is same as linear transformation like Fourier transformation with one difference, it allow time localization of different frequency components i.e., Wavelet transformation decomposes the signal in time and frequency domain.

There are two categories in the wavelet transformation.

i) Continuous Wavelet Transform.

ii) Discrete Wavelet Transform.

The continuous wavelet transform of a signal is defined as

$\operatorname{CWT}(\mathrm{y}, \mathrm{p}, \mathrm{g})=\frac{1}{\sqrt{|\mathrm{a}|}} \int \mathrm{y}(\mathrm{t}) \varphi_{\mathrm{p}, \mathrm{g}}^{*} \frac{(\mathrm{t}-\mathrm{g})}{\mathrm{p}} \mathrm{dt}$

Where $\varphi(\mathrm{t})$ is called as mother wavelet.

Where $\mathrm{p}$ and $\mathrm{q}$ are the constants. The constant $\mathrm{p}$ is called as dilation parameter and gis called as translation parameters. CWT $(y, p, g)$ denotes wavelets transform of the signal y with scale(dilation) denoted by p, times shift ( translation) denoted by q.

The discrete wavelet transform of the signal is defined as $\operatorname{DWT}(\mathrm{y}, \mathrm{k}, \mathrm{l})=\frac{1}{\sqrt{|\mathrm{a}|}} \int \mathrm{y}(\mathrm{m}) \varphi_{\mathrm{k}, \mathrm{l}}^{*}\left(1-\mathrm{ka}^{\mathrm{k}}{ }_{\mathrm{o}}\right) /\left(\mathrm{a}_{\mathrm{o}}^{\mathrm{k}}\right) \varphi \mathrm{dt}$ 
Here $\mathrm{a}_{0}{ }^{\mathrm{k}}$ and $\mathrm{la}_{\mathrm{o}}{ }^{\mathrm{k}}$ are dilated and translated parameters. $\mathrm{k}$ and $\mathrm{l}$ are the integer variables.

During the occurrence of the fault the current and voltage can undergo transients and these can be analysed using discrete wavelet transform. Normally the implementation of DWT is done by mallets algorithm and its formulation is related to the filter bank theory. Wavelet transformation techniques use 2 filters HP which is nothing but detail coefficient and LP which is approximation coefficient. From mother wavelets HP is derived and from scaling function which is associated with mother wavelet LP is derived.

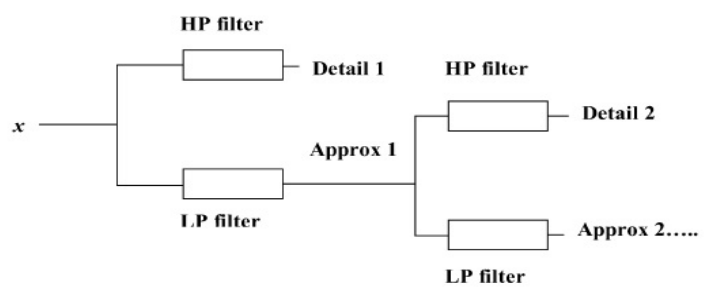

Figure 1: shows implementation of MRA using Filter bank theory.

\section{FAULT CLASSIFICATION ALGORITHM}

The most important tool for digital relaying system is wavelet transform. The classification algorithm for transmission line is done by using wavelet MRA(Multi resolution technique) are based on measurement and comparison of sharp signal variation in the current value for three phase in the first stage MRA (Multi resolution technique) detail signal extracted from original fault signal[1][4]. The simulation model of three phase power system has been simulated. The lines current is processed using WT (wavelet transform) classification algorithm to obtain wavelet MRA coefficient. For fault classification these coefficients are used.

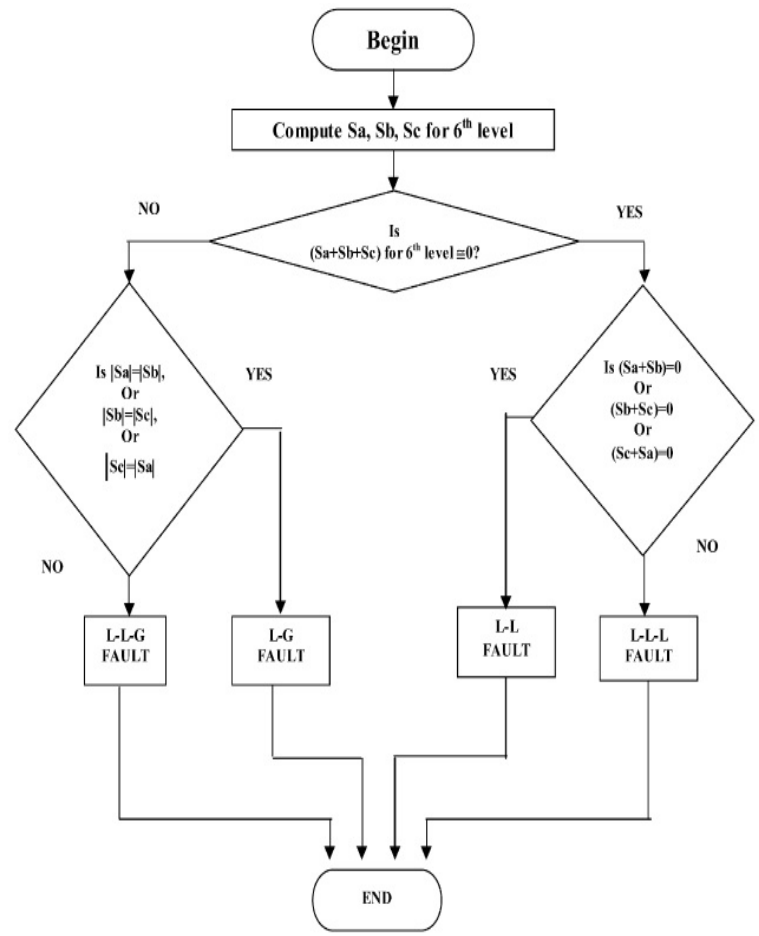

Figure 2: The Fault Classification algorithm.

Let us Consider $\mathrm{S}_{\mathrm{ph}-\mathrm{a}}, \mathrm{S}_{\mathrm{ph}-\mathrm{b}} \mathrm{S}_{\mathrm{ph}-\mathrm{c}}$ are the sum of 6 th level detail coefficient values for current in phase a, phase $\mathrm{b}$, phase $\mathrm{c}$. When summation of $S_{\text {ph-a }}, S_{\text {ph-b }} S_{\text {ph-c }}$ is nearly equal to 0 then the fault can be either three phase fault or L-L fault. If the summation of any two phases tends to or nearly equal to 0 then it is L-L fault. If the $S_{\text {ph-a }}, S_{\text {ph-b }}$ and $S_{\text {ph-c }}$ be the summation of sixth level detail coefficient values is not equal to 0 then it is L-G(line to ground) or L-L-L-G(three phase) fault. If the summations of magnitude of any two phases are equal then it is L-G fault.

At normal condition the three phases current were recorded and decomposed using DWT to get maximum detailed coefficient.Db4 (Daubechies wavelet 4) is used as mother wavelets and $12.5 \mathrm{kHz}$ sampling rate is selected[1]. Only 6th level output is considered among different decomposition level for the analysis of fault classification. Summation of sixth level output d6 gives the frequency components which corresponding to second and third harmonics. 


\section{SIMULATION AND RESULT}

Consider a transmission line having a length $300 \mathrm{~km}$ with $400 \mathrm{kVas}$ three phase source voltage. The three phase mutual inductance positive(+ve) sequence parameters values are resistance $2.34 \Omega$, inductance $95.10 \mathrm{mH}$, and capacitance 1.24 $\mu \mathrm{F}$ for each hundred kilometres length. The zero sequence line parameters are resistance $38.85 \Omega$, inductance 325.08 $\mathrm{mH}$ and capacitance $0.845 \mu \mathrm{F}$ for each hundred kilometres length. The negative (-ve) sequence line parameters and positive sequence parameters are same. The source impedance $(\mathrm{Zs})$ for positive and negative sequence of are $(0.45+\mathrm{j}$ $5) \Omega$ per phase. The zero sequence impedance is one and half times the positive sequence impedance. At the load side $500 \mathrm{MW}$ an active power and inductive reactive power of 20 MVAR have been considered. The simulation result for different type of faults is shown in the below figure.

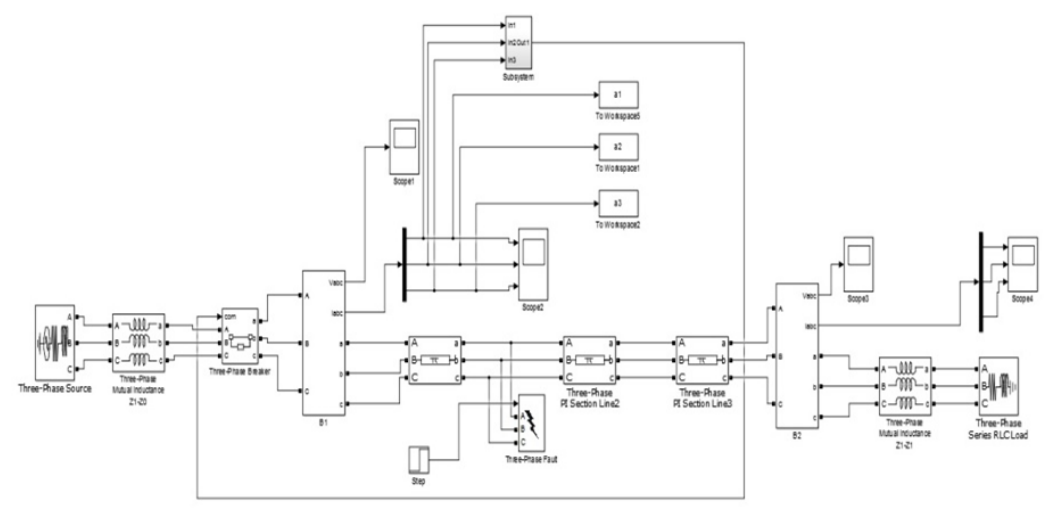

Figure 3: Shows the power system simulation block.

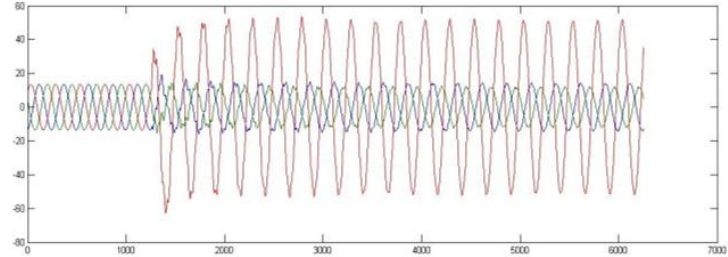

Figure 4.1: Simulation result for L-G faults.

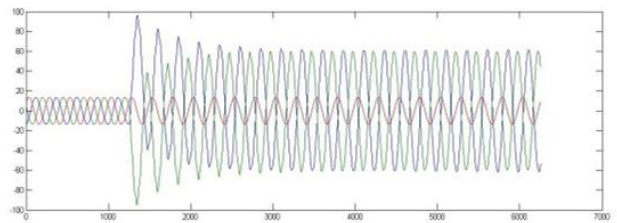

Figure 4.3: Simulation result for L-L faults.

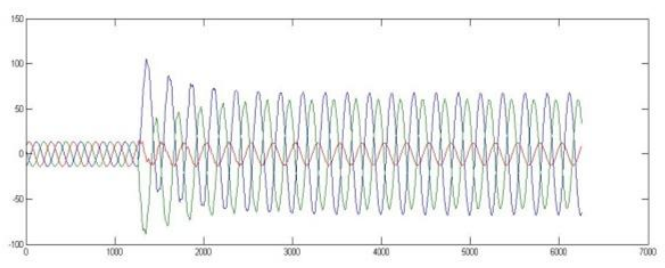

Figure 4.2: Simulation result for L-L-G faults.

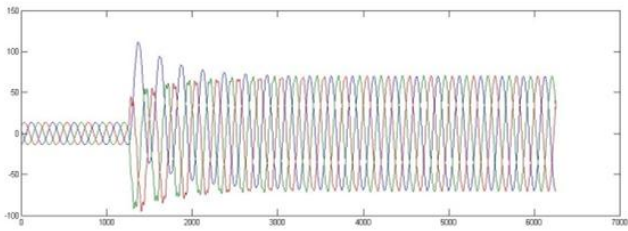

Figure 4.4. Simulation result for L-L-L faults.

Simulation is carried out by using MATLAB/SIMULINK with different types of faults. The summation for different phases is shown in the table.

The below tabulation values shows the summation of all three phase values by using DB4 as mother wavelets.

\begin{tabular}{|c|c|c|c|c|c|c|c|}
\hline $\begin{array}{l}\text { FAULTS } \\
\text { TYPE }\end{array}$ & $\mathrm{S}_{\mathrm{ph}-\mathrm{s}}$ & $\mathrm{S}_{\mathrm{ph}-\mathrm{b}}$ & $\mathrm{S}_{\mathrm{ah}-\mathrm{c}}$ & $\begin{array}{c}\mathrm{S}_{\mathrm{ph}-\mathrm{s}}+\mathrm{S}_{\mathrm{ph}-\mathrm{b}}+\mathrm{S}_{\mathrm{ph}-} \\
\mathrm{c} \approx 0 \\
(\mathrm{Yes} \backslash \mathrm{No}) \\
\end{array}$ & $\begin{array}{l}\mathrm{S}_{\mathrm{d} p \mathrm{~h}-\mathrm{a}}+\mathrm{S}_{\mathrm{ph} \mathrm{h}-\mathrm{b}} \approx 0 \\
\quad(\mathrm{Yes} \backslash \mathrm{No})\end{array}$ & $\begin{array}{c}\mathrm{S}_{\mathrm{ph}-\mathrm{b}}+\mathrm{S}_{\mathrm{ph}-\mathrm{c}} \\
\approx 0 \\
(\mathrm{Yes} \backslash \mathrm{No})\end{array}$ & $\begin{array}{c}\mathrm{S}_{\text {ph-s }}+\mathrm{S}_{\text {ph-c }} \\
\approx 0 \\
(\mathrm{Yes} \backslash \mathrm{No}) \\
\end{array}$ \\
\hline A B & -60.5808 & 68.8693 & -8.37314 & Yes & Yes & No & No \\
\hline $\mathrm{BC}$ & -6.477 & 106.7986 & -100.12 & Yes & No & Yes & No \\
\hline $\mathrm{AC}$ & 31.25034 & 14.85014 & -46.2179 & Yes & No & No & Yes \\
\hline $\mathrm{ABC}$ & -17.3273 & 112.1228 & -94.7955 & Yes & No & No & No \\
\hline $\begin{array}{l}\text { FAULTS } \\
\text { TYPE }\end{array}$ & $\mathrm{S}_{\mathrm{ph}-\mathrm{a}}$ & $\mathrm{S}_{\mathrm{ph}-\mathrm{b}}$ & $\mathrm{S}_{\mathrm{ph}-\mathrm{c}}$ & $\begin{array}{c}\mathrm{S}_{\mathrm{ph}-\mathrm{s}}+\mathrm{S}_{\mathrm{ph}-\mathrm{b}}+\mathrm{S}_{\mathrm{ph}-} \\
\mathrm{c} \approx 0 \\
(\mathrm{Yes} \backslash \mathrm{No}) \\
\end{array}$ & $\left|\mathbf{S}_{\mathrm{g} \mathrm{bh}-\mathrm{a}}\right| \approx\left|\mathrm{S}_{\mathrm{ch}-\mathrm{b}}\right|$ & $\begin{array}{c}\left|\mathrm{S}_{\mathrm{ph}-\mathrm{b}}\right| \approx \mid \mathrm{S}_{\mathrm{ph}-} \\
\mathrm{d}\end{array}$ & $\left|\mathrm{S}_{\mathrm{ph}-\mathrm{a}}\right| \approx\left|\mathrm{S}_{\mathrm{ph}-\mathrm{c}}\right|$ \\
\hline $\mathrm{AG}$ & -26.227 & 16.30631 & -6.93544 & No & No & Yes & No \\
\hline BG & -3.91933 & 85.16764 & -6.08014 & No & No & No & Yes \\
\hline $\mathrm{CG}$ & -10.2262 & 10.85478 & -58.9406 & No & Yes & No & No \\
\hline BCG & -7.14047 & 115.8345 & -91.0838 & No & No & No & No \\
\hline ACG & 4.46656 & 12.39344 & -73.0016 & No & No & No & No \\
\hline $\mathrm{ABG}$ & -42.8328 & 86.61722 & -5.25297 & No & No & No & No \\
\hline
\end{tabular}

Table 1.1:Tabulation of fault classification algorithm using Db4 as mother wavelet. 
The below tabulation value shows the summation of all three phase values by using SYM4 as mother wavelets.

\begin{tabular}{|c|c|c|c|c|c|c|c|}
\hline $\begin{array}{l}\text { FAULTS } \\
\text { TYPE }\end{array}$ & $\mathrm{S}_{\mathrm{ghl-a}}$ & $\mathrm{S}_{\mathrm{ghl}-\mathrm{b}}$ & $S_{\text {ohl-c }}$ & $\begin{array}{c}\mathrm{S}_{\mathrm{ph}-\mathrm{a}}+\mathrm{S}_{\mathrm{ph}-\mathrm{b}}+\mathrm{S}_{\mathrm{ph}-} \\
\mathrm{c} \approx 0 \\
(\mathrm{Yes} \backslash \mathrm{No}) \\
\end{array}$ & $\begin{array}{c}\mathrm{S}_{\mathrm{ph}-\mathrm{a}}+\mathrm{S}_{\mathrm{ph}-\mathrm{b}} \\
\approx 0 \\
(\mathrm{Yes} \backslash \mathrm{No}) \\
\end{array}$ & $\begin{array}{c}\mathrm{S}_{\mathrm{ph}-\mathrm{b} \mathrm{h}}+\mathrm{S}_{\mathrm{ph}-\mathrm{c}} \approx 0 \\
(\mathrm{Yes} \backslash \mathrm{No})\end{array}$ & $\begin{array}{c}\mathrm{S}_{\mathrm{ph}-\mathrm{q}}+\mathrm{S}_{\mathrm{ph}-\mathrm{c}} \\
\approx 0 \\
(\mathrm{Yes} \backslash \mathrm{No}) \\
\end{array}$ \\
\hline $\mathrm{AB}$ & 5.077707 & -41.3039 & 36.23972 & No & No & Yes & No \\
\hline $\mathrm{BC}$ & -8.08564 & -65.5145 & 73.52014 & No & No & Yes & No \\
\hline $\mathrm{AC}$ & -32.2162 & -28.1541 & 60.43681 & No & No & No & No \\
\hline $\mathrm{ABC}$ & -15.4238 & -61.8054 & 77.22922 & No & No & No & No \\
\hline $\begin{array}{l}\text { FAULTS } \\
\text { TYPE }\end{array}$ & $\mathrm{S}_{\mathrm{ph}-\mathrm{a}}$ & $\mathrm{S}_{\mathrm{ph}-\mathrm{b}}$ & $\mathrm{S}_{\mathrm{ph}-\mathrm{c}}$ & $\begin{array}{c}\mathrm{S}_{\mathrm{ph}-\mathrm{a}}+\mathrm{S}_{\mathrm{ph}-\mathrm{b}}+\mathrm{S}_{\mathrm{ph}-} \\
\approx \approx 0 \\
(\mathrm{Yes} \backslash \mathrm{No})\end{array}$ & $\left|\mathrm{S}_{\mathrm{ph}-\mathrm{s}}\right| \approx\left|\mathrm{S}_{\mathrm{ph}-\mathrm{b}}\right|$ & $\left|\mathrm{S}_{\mathrm{ph}-\mathrm{b}}\right| \approx\left|\mathrm{S}_{\mathrm{ph}-\mathrm{c}}\right|$ & $\left|\mathrm{S}_{\mathrm{ph}-\mathrm{s}}\right| \approx\left|\mathrm{S}_{\mathrm{ph}-\mathrm{c}}\right|$ \\
\hline $\mathrm{AG}$ & -7.7941 & -29.0794 & 35.293 & No & No & Yes & No \\
\hline BG & -8.53916 & -53.93 & 35.87968 & No & No & No & No \\
\hline $\mathrm{CG}$ & -6.80356 & -26.7538 & 61.72406 & No & No & No & No \\
\hline $\mathrm{BCG}$ & -7.50736 & -66.3741 & 72.66056 & No & No & Yes & No \\
\hline ACG & -22.2648 & -27.736 & 70.38826 & No & No & No & No \\
\hline $\mathrm{ABG}$ & -4.01416 & -50.3958 & 35.24334 & No & No & No & No \\
\hline
\end{tabular}

Table 1.2: Tabulation of fault classification algorithm using sym4 as mother wavelet.

\begin{tabular}{|c|c|c|c|c|c|c|c|}
\hline $\begin{array}{c}\text { FAULTS } \\
\text { TYPE }\end{array}$ & $\mathrm{S}_{\mathrm{gh}-\mathrm{a}}$ & $\mathrm{S}_{\mathrm{kh-b}}$ & $\mathrm{S}_{\mathrm{ph}-\mathrm{c}}$ & $\begin{array}{c}\mathrm{S}_{\mathrm{ph}-\mathrm{a}}+\mathrm{S}_{\mathrm{ph}-\mathrm{b}}+\mathrm{S}_{\mathrm{ph}-} \\
\mathrm{c} \approx 0 \\
(\mathrm{Yes} \backslash \mathrm{No})\end{array}$ & $\begin{array}{c}\mathrm{S}_{\text {ph-s }}+\mathrm{S}_{\mathrm{ph}-\mathrm{b}} \approx 0 \\
(\mathrm{Yes} \backslash \mathrm{No})\end{array}$ & $\begin{array}{c}\mathrm{S}_{\text {ph-b }}+\mathrm{S}_{\text {ph-c }} \\
\approx 0 \\
(\mathrm{Yes} \backslash \mathrm{No}) \\
\end{array}$ & $\begin{array}{c}\mathrm{S}_{\text {ph-s }}+\mathrm{S}_{\text {ph-c }} \\
\approx 0 \\
(\mathrm{Yes} \backslash \mathrm{No})\end{array}$ \\
\hline $\mathrm{AB}$ & -20.3609 & 12.78457 & 7.561724 & Yes & No & No & No \\
\hline $\mathrm{BC}$ & -10.2402 & 24.27165 & -13.9839 & Yes & No & No & No \\
\hline $\mathrm{AC}$ & 1.199295 & 2.678429 & -3.91074 & Yes & No & No & No \\
\hline $\mathrm{ABC}$ & -9.34515 & 23.80033 & -14.4552 & Yes & No & No & No \\
\hline $\begin{array}{c}\text { FAULTS } \\
\text { TYPE }\end{array}$ & $\mathrm{S}_{\mathrm{shh- \textrm {a }}}$ & $\mathrm{S}_{\mathrm{ph}-\mathrm{b}}$ & $\mathrm{S}_{\mathrm{ph}-\mathrm{c}}$ & $\begin{array}{c}\mathrm{S}_{\mathrm{ph}-\mathrm{a}}+\mathrm{S}_{\mathrm{ph}-\mathrm{b}}+\mathrm{S}_{\mathrm{ph}-} \\
\mathrm{c} \approx 0 \\
(\mathrm{Yes} \backslash \mathrm{No}) \\
\end{array}$ & $\left|\mathrm{S}_{\mathrm{ph}-\mathrm{a}}\right| \approx\left|\mathrm{S}_{\mathrm{ph}-\mathrm{b}}\right|$ & $\begin{array}{c}\left|\mathrm{S}_{\text {ph-b }}\right| \approx \mid \mathrm{S}_{\text {ph- }} \\
\mathrm{d}\end{array}$ & $\left|\mathrm{S}_{\mathrm{ph}-\mathrm{a}}\right| \approx\left|\mathrm{S}_{\text {ph-c }}\right|$ \\
\hline $\mathrm{AG}$ & -12.6961 & 3.91933 & 7.984315 & No & No & Yes & No \\
\hline BG & -10.0861 & 18.99571 & 7.659171 & No & Yes & No & No \\
\hline $\mathrm{CG}$ & -10.7541 & 2.101798 & -6.29965 & No & No & No & Yes \\
\hline BCG & -10.4876 & 25.62561 & -12.6299 & No & No & No & No \\
\hline $\mathrm{ACG}$ & -4.75641 & 2.18367 & -9.86645 & No & No & No & No \\
\hline $\mathrm{ABG}$ & -15.7592 & 17.38631 & 8.303919 & No & No & No & No \\
\hline
\end{tabular}

Table 1.3:Tabulation of fault classification algorithm using Coif4 as mother wavelet.

The above tabulation value shows the summation of all three phase values by using Coif 4 as mother wavelets.

\begin{tabular}{|c|c|c|c|c|c|c|c|}
\hline $\begin{array}{l}\text { FAULT } \\
\text { S TYPE }\end{array}$ & $\mathrm{S}_{\mathrm{shh- \textrm {s }}}$ & $S_{s h-b}$ & $S_{\text {sh-c }}$ & 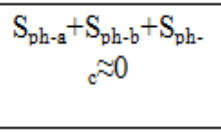 & $\begin{array}{l}\mathrm{S}_{\mathrm{ph}-\mathrm{s}}+\mathrm{S}_{\mathrm{ph}-\mathrm{b}} \approx 0 \\
\quad \text { (Yes }(\mathrm{No})\end{array}$ & $\begin{array}{l}\mathrm{S}_{\text {ph-b }}+\mathrm{S}_{\text {ph-c }} \approx 0 \\
\quad(\text { Yes } N \text { No) }\end{array}$ & $\begin{array}{c}\mathrm{S}_{\mathrm{ph}-\mathrm{a}}+\mathrm{S}_{\mathrm{phh}-\mathrm{c}} \\
\approx 0 \\
(\mathrm{Yes} \backslash \mathrm{No}) \\
\end{array}$ \\
\hline $\mathrm{AB}$ & -150.744 & 201.6453 & -51.0528 & Yes & No & No & No \\
\hline $\mathrm{BC}$ & -43.0129 & 311.8393 & -268.361 & Yes & No & No & No \\
\hline $\mathrm{AC}$ & 66.71542 & 94.06565 & -161.095 & Yes & No & No & No \\
\hline $\mathrm{ABC}$ & -41.5264 & 310.8633 & -269.337 & Yes & No & No & No \\
\hline $\begin{array}{l}\text { FAULT } \\
\text { S TYPE }\end{array}$ & $S_{\text {shl-s }}$ & $\mathrm{S}_{\mathrm{sh-b}}$ & $\mathrm{S}_{\mathrm{wh-c}}$ & $\begin{array}{c}\mathrm{S}_{\mathrm{ph}-\mathrm{s}}+\mathrm{S}_{\mathrm{ph}-\mathrm{b}}+\mathrm{S}_{\mathrm{ph}-} \\
\approx \approx 0\end{array}$ & $\left|S_{\mathrm{ph}-\mathrm{a}}\right| \approx\left|\mathbf{S}_{\mathrm{sh-b}-\mathrm{b}}\right|$ & $\left|\mathrm{S}_{\mathrm{ph}-\mathrm{s}}\right| \approx\left|\mathrm{S}_{\mathrm{ph}-\mathrm{b}}\right|$ & $\begin{array}{c}\left|\mathrm{S}_{\mathrm{ph}-\mathrm{s}}\right| \approx \mid \mathrm{S}_{\mathrm{ph}} \\
b \mid\end{array}$ \\
\hline $\mathrm{AG}$ & -71.8243 & 98.37661 & -46.7138 & No & No & No & No \\
\hline BG & -37.8507 & 255.0133 & -46.4728 & No & No & No & No \\
\hline CG & -51.9038 & 84.5645 & -183.189 & No & No & No & No \\
\hline $\mathrm{BCG}$ & -45.2263 & 326.4593 & -253.741 & No & No & No & No \\
\hline $\mathrm{ACG}$ & 5.234058 & 8.35471 & -222.576 & No & No & No & No \\
\hline $\mathrm{ABG}$ & -15.7592 & 248.5067 & -43.1284 & No & No & No & No \\
\hline
\end{tabular}

Table 1.4: Tabulation of fault classification algorithm using Bior1.3 as mother wavelet.

The above tabulation value shows the summation of all three phase values by using Bior1.3 as mother wavelets. 


\section{CONCLUSION}

Algorithm used for fault classification using $\mathrm{Db} 4$ wavelets for a distance of $100 \mathrm{~km}$ from the source considering all type of faults is assumed to be effective and yielded to be right classification. Simulation is carried out for different standard wavelets and only $\mathrm{Db} 4$ wavelet give accurate and exact fault classification when considering all other wavelets.

\section{ACKNOWLEDGEMENTS}

I would like to give my sincere gratitude to VISVESVARAYA TECHNOLOGICAL UNIVERSITY and NMAM INSTITUTE OF TECHNOLOGY for all support and encouragement.

\section{REFERENCES}

[1]. Jaya Bharata Reddy M, Mohanta DK. A DSP based frequency domain approach for classification of transmission line faults. Int J Digit Signal Process 2008;18(5).

[2]. D. Chanda, N.K. Kishore, A.K. Sinha, Application of wavelet multiresolution analysis for classification of faults on transmission lines, in:IEEE Conference on Convergent. Technologies for Asia-Pacific Region, vol. 4, 2003, pp. 14641469.

[3]. Wavelet toolbox, Matlab 7.0, The Math Works Inc., http://www.mathworks.com.

[4]. Mallat, S.G. A theory for multiresolution signal decomposition: the wavelet representation, IEEE Transactions on pattern analysis and machine intelligence, Vol. 11, No 7,Jul. 1989, pp. 674-693.

[5]. R.N. Mahanty, P.B. Dutta Gupta, An improved method for digital relaying of transmission lines, Electr. Power Compon Syst. 32 (2004) 10131030.

[6]. Yellaji Allipilli and G.Narasimha Rao, Detection and Classification of Faults in Transmission Lines Based on Wavelets, International Conference on Electrical, Electronics, Signals, Communication and Optimization (EESCO) - 2015. 